\title{
Comparaison de la dérive pour deux types de pulvérisateurs utilisés en production cotonnière au Bénin
}

\author{
Abdoul-Ibrachi Gouda ${ }^{(1,2)}$, Mahodjègbé Hermas Luc Mehoba ${ }^{(2)}$, Ibrahim Imorou Toko ${ }^{(1)}$, \\ Marie-Louise Scippo ${ }^{(3)}$, Patrick Kestemont ${ }^{(4)}$, Bruno Schiffers ${ }^{(2)}$ \\ (1) Université de Parakou. Faculté d'Agronomie. Département des Sciences et Techniques de Production Animale. \\ Laboratoire de Recherche en Aquaculture et Écotoxicologie Aquatique. BP 123. Parakou (Bénin). \\ E-mail : goudaibrachi@yahoo.fr \\ (2) Université de Liège - Gembloux Agro-Bio Tech. Laboratoire de Phytopharmacie. Passage des Déportés, 2. \\ BE-5030 Gembloux (Belgique). \\ (3) Université de Liège. Faculté de Médecine vétérinaire. Laboratoire d'Analyse des Denrées Alimentaires. \\ Boulevard de Colonster, 20. Bât B43B. Sart-Tilman. BE-4000 Liège (Belgique). \\ (4) Université de Namur. Faculté des Sciences. Unité de Recherche en Biologie Environnementale et Évolutive. \\ Rue de Bruxelles, 61. BE-5000 Namur (Belgique).
}

Reçu le 8 mai 2017, accepté le 16 avril 2018, mis en ligne le 8 mai 2018.

Cet article est distribué suivant les termes et les conditions de la licence CC-BY (http://creativecommons.org/licenses/by/4.0/ deed.fr)

Description du sujet. L'utilisation accrue des produits phytosanitaires et la variation des substances actives ces dernières années soulèvent des interrogations sur le niveau de pollution de l'environnement et celui des écosystèmes aquatiques du bassin cotonnier en particulier.

Objectifs. Des essais ont été menés (à Batran, commune de Banikoara) en conditions pratiques pour quantifier et comparer la dérive engendrée lors des pulvérisations effectuées avec la canne centrifuge et le pulvérisateur à dos, deux appareils spécifiquement utilisés dans le bassin cotonnier au Bénin.

Méthode. Une bouillie de tartrazine (un colorant alimentaire) à $20 \mathrm{~g} \cdot \mathrm{l}^{-1}$ a été utilisée. Dix essais de pulvérisation à des hauteurs de $1 \mathrm{~m}$ et de 1,5 $\mathrm{m}$ ont été réalisés suivant un dispositif de collecte préétabli et validé par un essai préalable avec des papiers hydrosensibles. Cinquante-quatre patchs de tissus coton blancs ont été placés à des hauteurs déterminées et à des distances régulières à partir du point d'émission. En fin d'essai, ils ont été récupérés et la concentration en tartrazine $\left(\mathrm{en} \mu \mathrm{g} \cdot \mathrm{cm}^{-2}\right)$ a été déterminée après extraction à l'eau à l'aide d'un colorimètre. À partir des quantités de tartrazine récupérées, les pourcentages de dérive ont été calculés, permettant de comparer la dérive engendrée par chaque appareil.

Résultats. Il apparait que, dans les mêmes conditions météorologiques $\left(35^{\circ} \pm 1{ }^{\circ} \mathrm{C}\right.$; H.R. : $64 \pm 4 \%$; vent stable d'environ $\left.3 \mathrm{~m} \cdot \mathrm{s}^{-1}\right)$, la hauteur de pulvérisation de $1,5 \mathrm{~m}$ et la canne de pulvérisation centrifuge engendrent des pourcentages de dérive significativement supérieurs à ceux générés respectivement pour la hauteur de pulvérisation de $1 \mathrm{~m}$ et le pulvérisateur à dos.

Conclusions. Quel que soit l'appareil, les gouttelettes se dispersent jusqu'à une distance de $16 \mathrm{~m}$.

Mots-clés. Pesticides, dérive, pulvérisateur, coton, Bénin.

\section{Comparison of drift of two types of sprayers used in cotton production in Benin}

Description of the subject. Increasing use of plant protection products and a continuous change in their active substances during recent years raise crucial questions regarding environmental pollution, particularly for aquatic ecosystems in the cotton production area.

Objectives. Field trials were carried out (in Batran, province of Banikoara) in conditions close to those of local practices. The objective was to quantify and compare the drift generated during spraying both with a centrifugal cane and a backpack sprayer, which are the two most popular types of sprayer used in the cotton area of Benin.

Method. A mixture of water and tartrazine (a food grade dye) was prepared for spraying at $20 \mathrm{~g} \cdot \mathrm{l}^{-1}$. Ten spraying trials were conducted, at two heights $(1 \mathrm{~m}$ and $1.5 \mathrm{~m})$, according to a pre-established protocol validated by an initial trial carried out with water-sensitive paper. Fifty-four white cotton patches were placed at the predetermined heights and distances from the 
emission point. After each trial, the patches were collected and the tartrazine concentrations (in $\left.\mu \mathrm{g} \cdot \mathrm{cm}^{-2}\right)$ were determined after extraction in water with a colorimeter. The percentages of drift were then calculated from the quantities of tartrazine recovered.

Results. Results showed that, in the same weather conditions $\left(35^{\circ} \pm 1{ }^{\circ} \mathrm{C}\right.$; R.H.: $64+4 \%$; wind speed stable around $\left.3 \mathrm{~m} \cdot \mathrm{s}^{-1}\right)$, spraying at the height of $1.5 \mathrm{~m}$ with the centrifugal cane generated drift percentages significantly higher than those generated by spraying at the height of $1 \mathrm{~m}$ with the backpack sprayer.

Conclusions. Droplets of mixture were observed at up to $16 \mathrm{~m}$, whichever sprayer was used.

Keywords. Pesticides, spray drift, sprayer, cotton, Benin.

\section{INTRODUCTION}

Le coton joue un rôle important dans la croissance économique du Bénin, avec une contribution de $32 \%$ au produit intérieur brut, représentant $90 \%$ des recettes d'exportations, $45 \%$ des rentrées fiscales hors douane et $60 \%$ du tissu industriel (MAAF, $2013^{1}$ cité par Zoumenou et al., 2015). Au Bénin, les recettes tirées de la culture du coton contribuent à la réalisation de nombreuses infrastructures socio-communautaires (AIC, 2005) et la filière bénéficie de ce fait du soutien des pouvoirs publics (Matthess et al., 2005). C'est pourquoi, malgré la forte pression parasitaire, chaque année au Bénin des centaines de milliers d'hectares (environ 400000 ha en 2015, selon la Société Nationale pour la Promotion Agricole) sont cultivés en coton (Celini, 2001) et, en moyenne, font l'objet de six traitements insecticides durant la saison (Djihinto et al., 2016).

Cependant, l'utilisation de ces produits phytopharmaceutiques dans les conditions habituellement rencontrées en Afrique de l'Ouest suscite de nombreuses inquiétudes, liées notamment à leur toxicité et à leur impact négatif pour l'homme et pour l'environnement (Sanborn et al., 2004 ; Toé et al., 2004 ; Démbélé, 2006 ; Kodjo, 2007 ; Bonicelli et al., 2012 ; Eddaya et al., 2015). Les insecticides et les herbicides, sélectionnés avant tout pour leur efficacité, sont pour la plupart très toxiques pour les opérateurs ou susceptibles de générer des effets indésirables, voire irréversibles, après exposition par voie dermale ou par inhalation (Adechian et al., 2015). Plusieurs auteurs estiment aussi que les risques pour l'environnement sont souvent ignorés ou minorés (Doumbia \& Kwadjo, 2009; Sougnabe et al., 2009; Thiam \& Sagna, 2009). Actuellement, au Bénin, plusieurs insecticides d'origines diverses et de qualité douteuse sont utilisés dans le bassin cotonnier. Le Ministère béninois de l'Agriculture, de l'Élevage et de la Pêche a notamment

MAAF (Ministère de l'Agriculture, de l'Agroalimentaire et de la Forêt), 2013. Les politiques agricoles à travers le monde. $4^{\mathrm{e}}$ éd. Paris : MAAF. recensé l'emploi d'insecticides organophosphorés (diméthoate, profenofos, chlorpyriphos, etc.), souvent utilisés en association avec des pyréthrinoïdes (perméthrine, cyperméthrine, bifenthrine, lambdacyhalothrine, etc.), des néonicotinoïdes (comme l'acétamipride) ou bien d'autres substances actives (indoxacarb, spinetoram, l'émamectine benzoate, etc.) (MAEP, 2016).

Par ailleurs, lors des traitements phytosanitaires, une partie de la bouillie n'atteint pas la cible et ces derniers génèrent donc des transferts (ou « dérive ») non négligeables de substances toxiques dans l'environnement (Herbst, 2001). Quelle que soit la méthode utilisée pour l'application des produits phytosanitaires, les pertes vers le milieu naturel ou d'autres espaces non visés, tels que les cultures adjacentes, seront plus ou moins importantes (Gil, 2007). Selon Bylemans (2001), la dérive explique une bonne partie de ces pertes. Plusieurs auteurs ont mené des études sur les principaux paramètres qui influencent la dérive (Stainier, 2004; Siati, 2005 ; Somerhausen, 2006 ; Reichenberger et al., 2007). Les plus importants sont la taille, la vitesse et la direction d'éjection des gouttes (Solie \& Alimardani, 1986 ; Qi et al., 2008) qui varient en fonction des appareils et des conditions d'emploi, les conditions météorologiques externes (notamment la vitesse du vent) (Gil \& Sinfort, 2005), et enfin la hauteur de la buse par rapport au sol (Baetens et al., 2009). Plusieurs auteurs ont ainsi montré une réduction significative de la dérive pour des gouttelettes de diamètre supérieur à $100 \mu \mathrm{m}$ (Matthews, 1992; Miller \& Butler Ellis, 2000). Le transport des gouttelettes loin des cibles est provoqué par les mouvements d'air (BCPC, 1986 ; Al Heidary et al., 2014). Briand et al. (2001) estiment que les pertes de bouillie par dérive peuvent représenter de $1 \%$ à $30 \%$ de la quantité pulvérisée, selon les circonstances d'application (volume de bouillie par ha, appareil mis en ouvre, type de buse, conditions climatiques, etc.).

Au Bénin, la proximité de retenues d'eau avec les champs cultivés et fréquemment traités est susceptible de générer des contaminations accidentelles par le ruissellement lors des épisodes pluvieux ou, lors des pulvérisations, par la dérive des gouttelettes entrainées 
par le vent vers la surface des eaux. Le risque de contamination des eaux est d'autant plus critique que les champs de coton sont installés aux environs immédiats de retenues d'eau ou de ruisseaux, malgré les recommandations des agents d'encadrement technique. Il en résulte une contamination potentielle des écosystèmes et des nuisances pour la santé humaine (Schilter, 1991 ; Kanda, 2011). Selon Orhon (1993), la contamination des eaux dépend de la distance séparant le lieu de pulvérisation du ruisseau collecteur. Or, Adechian et al. (2015) constatent que dans la zone du bassin cotonnier, $46 \%$ des producteurs ont un champ situé à moins de $500 \mathrm{~m}$ d'un cours d'eau ou d'un plan d'eau où sont produits des poissons. Le coton figurant parmi les spéculations les plus citées, les insecticides sont fréquemment utilisés aux abords des plans d'eau. Gbaguidi et al. (2011) ont ainsi relevé dans la commune de Savalou (Bénin) des concentrations dans les eaux de la rivière Agbado variant de 0,05 à $0,475 \mu \mathrm{g} \cdot 1^{-1}$ pour l'atrazine, de 0,10 à $1,316 \mu \mathrm{g} \cdot \mathrm{l}^{-1}$ pour le glyphosate et de 0,75 à $4,450 \mu \mathrm{g} \cdot l^{-}$pour des insecticides pyréthrinoïdes. Agbohessi (2014) signale des teneurs de 1 à $100 \mu \mathrm{g} \cdot \mathrm{l}^{-1}$ en DDT et de 58 à $746 \mu \mathrm{g} \cdot \mathrm{l}^{-1}$ en endosulfan pour l'eau de surface dans les zones cynégétiques du Pendjari, du Djona et du Parc National du W.

Au-delà du risque de contamination des eaux, se pose aussi (dans le cadre du projet ARES-AQUATOX) la question du risque d'exposition des consommateurs de la région via les poissons contaminés par les pesticides appliqués dans les champs (Sanborn et al., 2004 ; Pazou et al., 2006a; Pazou et al., 2006b). En effet, après un dosage des résidus de pesticide, Okoumassoun et al. (2002) signalent la présence du lindane $\left(105 \mu \mathrm{g} \cdot \mathrm{g}^{-1}\right)$, de la dieldrine $\left(75 \mu \mathrm{g} \cdot \mathrm{g}^{-1}\right)$, de l'heptachlore $\left(30 \mu \mathrm{g} \cdot \mathrm{g}^{-1}\right)$, du pp-TDE $\left(28 \mu \mathrm{g} \cdot \mathrm{g}^{-1}\right)$ chez les tilapias mâles (Sarotherodon melanotheron Rüppell, 1852) capturés le long du fleuve Ouémé, ainsi qu'une concentration plasmatique en vitellogénine atteignant $38 \mu \mathrm{g} \cdot \mathrm{ml}^{-1}$. En outre, une contamination de l'eau souterraine par les pesticides organophosphorés et organochlorés est également possible comme Traoré et al. (2006) l'ont démontré pour les régions agricoles de Côte d'Ivoire où sont cultivés le cacao, le café, la banane et les légumes.

Pour appréhender le risque de contamination du milieu par les pesticides appliqués sur les champs de coton, il était intéressant de quantifier et de comparer la dérive générée par les deux types de pulvérisateurs (la canne centrifuge et le pulvérisateur à dos, dont le diamètre des gouttes est très différent) qui sont les plus utilisés lors des traitements phytosanitaires dans le bassin cotonnier au Bénin, en réalisant des essais avec ces appareils dans les conditions de la pratique locale. Il sera alors possible de mieux estimer la part de la contamination des retenues d'eau due à la dérive par rapport aux transferts par ruissellement.

\section{MATÉRIEL ET MÉTHODES}

\subsection{Choix du site pour la réalisation des essais}

Le choix s'est porté sur la commune de Banikoara (département de l'Alibori, Nord du Bénin), une des plus productrices de coton au Bénin, contribuant à elle seule à près de $40 \%$ de la production nationale (plus de 60000 ha cultivés, avec un rendement moyen d'environ $950 \mathrm{~kg} \cdot \mathrm{ha}^{-1}$ ). Cette zone est caractérisée par une pluviométrie moyenne de $850 \mathrm{~mm}$ par an, une température moyenne de $27,5^{\circ} \mathrm{C}$ et une humidité relative moyenne de $50 \%$ (Katé et al., 2014). Dans cette commune, une utilisation intensive de pesticides chimiques a été observée (Agbohessi et al., 2011), principalement sur les cultures de coton. Les essais sur la dérive ont été menés dans le village de Batran qui possède une importante retenue d'eau autour de laquelle se développe aussi, en contre-saison, une production maraichère intensive, fortement consommatrice de pesticides. Par ailleurs, tout au long de l'année, la retenue de Batran fournit la commune en poisson grâce aux pêcheurs maliens et nigériens installés dans le village. Les essais ont été menés avec des producteurs de coton membres de la Coopérative Villageoise des Producteurs de Coton (CVPC) de Batran durant la campagne cotonnière 2015-2016.

\subsection{Mesure de la dérive avec deux types d'appareil d'application}

Pour les essais de mesure de la dérive, les appareils ont été manipulés par les producteurs locaux pour que l'opération de traitement soit aussi fidèle que possible aux conditions de travail usuelles, celles-ci étant le plus souvent très éloignées des recommandations des fabricants.

Dispositif expérimental. Dix producteurs ont été choisis aléatoirement dans le répertoire de la coopérative avec pour seule condition d'avoir au moins cinq années d'expérience professionnelle et de disposer de champs de coton situés à proximité de la retenue d'eau. Ils ont été répartis en deux groupes, pour réaliser respectivement cinq essais avec un pulvérisateur à dos à pression entretenue et cinq essais avec une canne de pulvérisation centrifuge (au total 10 essais). Chaque application de bouillie (de l'eau avec un colorant) est réalisée selon un protocole identique (seul le volume pulvérisé varie selon le type d'appareil employé et la compétence de l'opérateur) et représente un essai.

Le dispositif expérimental (Figure 1) est installé sur un terrain plat en absence de végétation (pour éviter toute interception des gouttelettes). Quel que soit l'appareil, la pulvérisation se fait sur une ligne et sur une distance de $40 \mathrm{~m}$ (appelée «zone de 


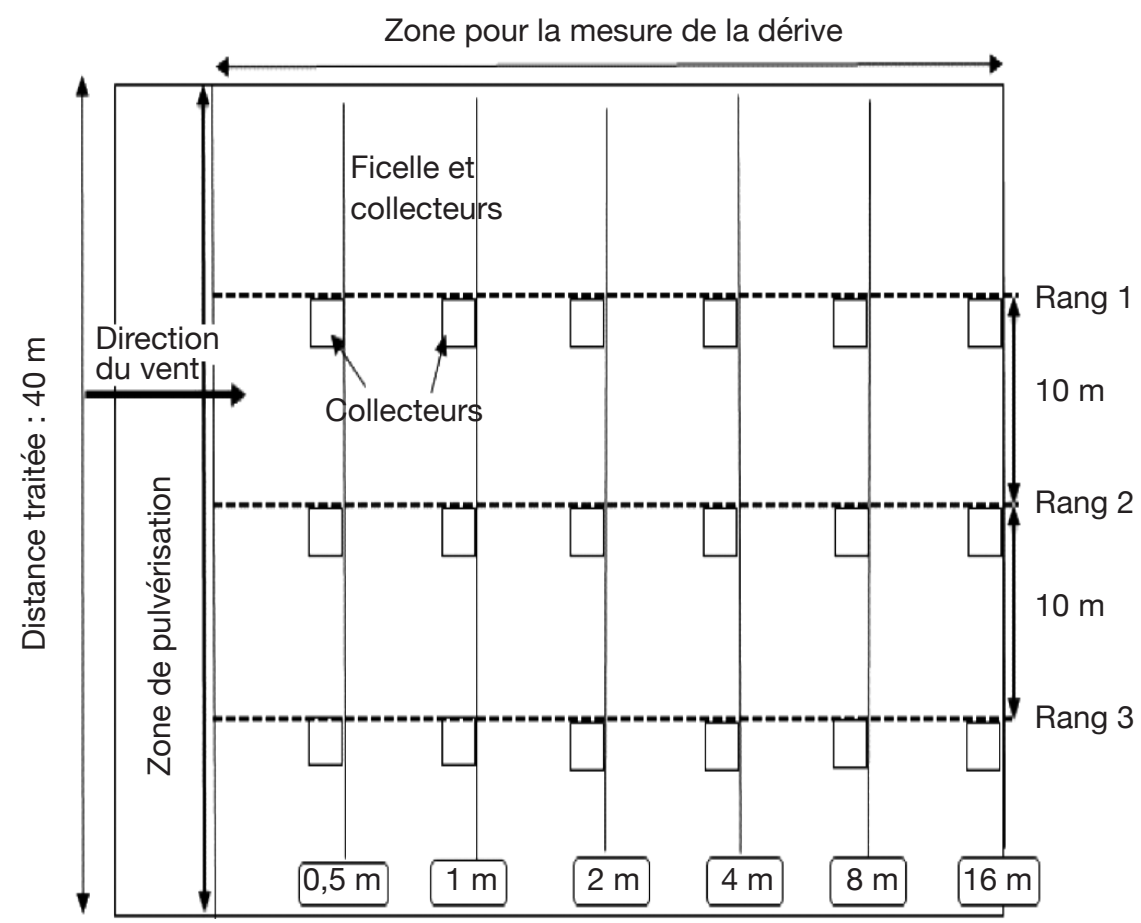

Figure 1. Schéma du dispositif d'essai de mesure de la dérive - Diagram of the measurement test device of the drift.

pulvérisation »), perpendiculairement à la direction du vent dominant qui entraine les gouttelettes vers des collecteurs placés sur trois rangs. Les collecteurs prévus pour absorber les gouttelettes qui contiennent le colorant sont constitués de carrés de $100 \mathrm{~cm}^{2}$ de coton blanc. Les collecteurs ont été disposés sur trois rangs, équidistants de $10 \mathrm{~m}$, perpendiculairement au sens de la pulvérisation. Sur base d'un essai préalable réalisé avec des papiers hydrosensibles (placés jusqu'à $32 \mathrm{~m}$ ), la distance maximale retenue pour quantifier la dérive est de $16 \mathrm{~m}$ à partir de la ligne d'application. Sur chaque rang, 18 collecteurs sont placés à différents intervalles (de $0,5 \mathrm{~m} ; 1 \mathrm{~m} ; 2 \mathrm{~m} ; 4 \mathrm{~m} ; 8 \mathrm{~m}$ ) jusqu'à $16 \mathrm{~m}$ de distance par rapport à la zone de pulvérisation. À chaque point de mesure, un collecteur est placé au sol, un sur une ficelle tendue à $0,5 \mathrm{~m}$ de hauteur et le dernier sur une ficelle à $1 \mathrm{~m}$ de hauteur. Les collecteurs placés au sol sont fixés horizontalement à l'aide de quatre aiguilles pour rester planes ; ceux placés en hauteur sont accrochés verticalement sur les ficelles à l'aide de pinces fixées à chaque extrémité pour éviter que leur surface ne soit concave ou convexe. Au total, 54 collecteurs (6 distances $\times 3$ hauteurs $\times 3$ rangs) sont ainsi répartis dans la zone de mesure de la dérive.

Pour chaque appareil, l'application a été répétée cinq fois à une hauteur de $1 \mathrm{~m}$ (représentant la hauteur moyenne habituelle du cotonnier lors des premiers traitements) et cinq fois à une hauteur de $1,5 \mathrm{~m}$ (représentant la croissance maximale du cotonnier). Durant les essais, chaque applicateur effectue 10 passages sur la zone de pulvérisation, suivant sa pratique et conformément à sa vitesse d'avancement habituelle qui a été enregistrée (chronométrage $\mathrm{du}$ temps mis pour effectuer les 10 passages) (Tableau 1). La vitesse moyenne d'avancement des producteurs utilisant la canne centrifuge a été plus rapide que celle de ceux qui utilisaient le pulvérisateur à dos (appareil plus volumineux et plus lourd). Au début de chaque essai, 11 de bouillie de tartrazine $\left(20 \mathrm{~g} \cdot \mathrm{l}^{-1}\right)$ est mise dans le réservoir de la canne de pulvérisation centrifuge et 31 dans celui du pulvérisateur à dos (le débit étant plus élevé). À la fin de l'essai, la quantité de bouillie utilisée est mesurée avec précision (par différence avec le volume restant dans le réservoir) afin d'estimer la quantité de tartrazine appliquée (Tableau 1).

Un thermo-hygromètre (TFA, Kat. Nr.30.5007) et un anémomètre (IHM CFM/CMM 6190 SI) ont servi pour la prise des données météorologiques. Durant les essais, la température de l'air a varié de $35 \pm 1{ }^{\circ} \mathrm{C}$ et l'humidité relative de $64 \pm 4 \%$. La vitesse moyenne du vent (mesurée dans les conditions habituelles locales de pulvérisation: zone libre de tout obstacle

Tableau 1. Paramètres enregistrés pour une application en fonction du type de pulvérisateur - Settings saved for an application according to the type of sprayer.

\begin{tabular}{lll}
\hline Paramètre & Pulvérisateur à dos & Canne centrifuge \\
\hline Vitesse moyenne d'avancement & $0,61 \pm 0,06 \mathrm{~m} \cdot \mathrm{s}^{-1}\left(2,19 \pm 0,23 \mathrm{~km} \cdot \mathrm{h}^{-1}\right)$ & $0,69 \pm 0,12 \mathrm{~m} \cdot \mathrm{s}^{-1}\left(2,47 \pm 0,42 \mathrm{~km} \cdot \mathrm{h}^{-1}\right)$ \\
$\begin{array}{l}\text { Temps moyen d'application } \\
\text { (durée moyenne d'un essai) }\end{array}$ & $664,1 \pm 2,68 \mathrm{~s}(11 \mathrm{~min} 4 \mathrm{~s} \pm 3 \mathrm{~s})$ & $598,1 \pm 4,67 \mathrm{~s}(9 \mathrm{~min} 58 \mathrm{~s} \pm 5 \mathrm{~s})$ \\
Volume moyen de bouillie épandu & $1,80 \pm 0,061$ & $0,55 \pm 0,131$ \\
Quantité moyenne de tartrazine épandue & $36 \pm 1,2 \mathrm{~g}$ & $11 \pm 2,6 \mathrm{~g}$ \\
\hline
\end{tabular}


et anémomètre tenu à bout de bras, à une hauteur d'environ $2 \mathrm{~m}$ ) est restée plus ou moins constante et égale à $3,0 \pm 0,4 \mathrm{~m} \cdot \mathrm{s}^{-1}\left(\right.$ soit $\left.10,8 \mathrm{~km} \cdot \mathrm{h}^{-1}\right)$, valeur qui est dans la fourchette recommandée pour la pulvérisation du coton avec ces appareils (Schiffers, 2011 ; Salah et al., 2015).

Matériel d'application. Deux appareils de pulvérisation, la canne de pulvérisation centrifuge à Ultra Bas Volume (QFG-15 LIMAO, appareil à piles) et le pulvérisateur à dos à pression entretenue (PIONEER 121 , équipé d'une buse à fente classique à jet pinceau, angle de $110^{\circ}$ ) réglé à 3 bars (débit mesuré de $\left.0,1791 \cdot \mathrm{min}^{-1}\right)$, ont été retenus pour les essais car ce sont ceux qui sont couramment utilisés par les producteurs de la région (au Bénin, la canne centrifuge étant plus souvent employée en coton que le pulvérisateur à dos, car son rendement de chantier est bien supérieur). Dans le cas de la canne centrifuge, la bouillie qui s'écoule par gravité est divisée en très fines gouttes (en moyenne de 70 à $150 \mu \mathrm{m}$ ) (Rachadi, 2010) à l'extrémité d'un disque dentelé qui tourne à très haute vitesse (environ 5000 tours $\cdot \mathrm{min}^{-1}$; éjection par la force centrifuge ; débit mesuré de $0,0631 \cdot \mathrm{min}^{-1}$ ). En ce qui concerne le pulvérisateur à dos, la bouillie est fragmentée en sortant sous pression par un ajutage calibré (une buse à fente, éjection sous la pression obtenue par la force mécanique exercée par un levier) : les gouttes sont significativement plus grosses pour cet appareil (en moyenne de 200 à $450 \mu \mathrm{m}$, sur base de la couleur de la buse utilisée, norme de l'American Society of Agricultural and Biological Engineers [ASABE]).

Préparation de la bouillie. La bouillie est constituée du mélange d'un traceur (la tartrazine, colorant alimentaire E102) en solution dans l'eau. La tartrazine a été choisie pour sa grande photostabilité, son absence de toxicité et sa bonne solubilité dans l'eau permettant de l'extraire facilement des collecteurs et d'en mesurer la concentration par colorimétrie. Selon Murray et al. (2000), dans les essais sur la dérive, la tartrazine à une concentration de $10 \mathrm{~g} \cdot \mathrm{l}^{-1}$ permet d'obtenir une bonne estimation du pourcentage de recouvrement et de la quantité déposée. Dans cette étude, étant donné les faibles volumes appliqués durant l'essai, une concentration de $20 \mathrm{~g} \cdot \mathrm{l}^{-1}$ a été utilisée afin de s'assurer de dépôts suffisants sur les collecteurs pour pouvoir être mesurés par colorimétrie après extraction.

Mesure quantitative des dépôts sur les collecteurs. La méthode de dosage par colorimétrie a été préalablement testée et validée dans une autre étude (Lawson et al., 2017). Après chaque essai, chacun des 54 collecteurs est récupéré et placé dans un tube FALCON® de $50 \mathrm{ml}$. Trente millilitres d'eau sont ajoutés et le tube est conservé pendant environ $12 \mathrm{~h}$ à température ambiante afin d'extraire le dépôt de tartrazine. Un colorimètre MN Nanocolor $500 \mathrm{D}$ a été utilisé pour la détermination de l'absorbance de la tartrazine extraite des collecteurs à la longueur d'onde de $436 \mathrm{~nm}$. L'absorbance à blanc a été mesurée sur deux collecteurs placés dans un tube FALCON ${ }^{\circledR}$ avec $30 \mathrm{ml}$ d'eau (blancos) pendant 12, 24 et $48 \mathrm{~h}$. Les valeurs d'absorbances obtenues pour les blancos peuvent être considérées comme négligeables (moyenne : 0,0015 après $48 \mathrm{~h}$ ). À partir d'une solution mère de tartrazine à $2800 \mu \mathrm{g} \cdot \mathrm{ml}^{-1}$, la droite de calibration a été réalisée pour six concentrations (de 0,44 à $14 \mu \mathrm{g} \cdot \mathrm{ml}^{-1}$ ). Les concentrations des extraits sont obtenues par calcul à partir de l'équation suivante :

$$
\mathrm{y}(\text { absorbance })=0,0561 * \mathrm{C}(\text { concentration })
$$

$\left(r^{2}=0,9995\right)$.

Traitement des données. Les données collectées ont été saisies et traitées à l'aide du tableur Excel et du logiciel Minitab 17. Ces logiciels ont permis de réaliser les statistiques descriptives utilisées pour calculer les moyennes arithmétiques et les écarts-types des dépôts pour chaque distance par rapport à la parcelle de pulvérisation.

\section{RÉSULTATS}

\subsection{Effet de la distance et de la hauteur de pulvérisation sur la dérive}

Les tableaux 2 et 3 reprennent les quantités de tartrazine mesurées sur les collecteurs (en $\mu \mathrm{g} \cdot \mathrm{cm}^{-2}$ ) après la pulvérisation avec chacun des deux appareils en fonction de la distance par rapport au point d'émission (de $0,5 \mathrm{~m}$ jusqu'à $16 \mathrm{~m}$ ), de la hauteur d'émission ( $1 \mathrm{~m}$ ou $1,5 \mathrm{~m}$ ) et du niveau de collecte (au sol, à $0,5 \mathrm{~m}$ et à $1 \mathrm{~m}$ ). Pour chaque niveau de collecte, la quantité de tartrazine indiquée correspond à la moyenne des mesures des cinq essais pour les trois rangs $(\mathrm{n}=15)$.

Pour chaque distance, la quantité moyenne de tartrazine est mesurée sur les collecteurs placés au sol (trois collecteurs) et en hauteur (six collecteurs). De plus, la quantité indiquée pour chaque distance correspond à la moyenne des mesures des cinq essais $(\mathrm{n}=45)$. Avec la canne centrifuge, elle varie de 7,27 \pm $2,57 \mu \mathrm{g} \cdot \mathrm{cm}^{-2}$ à $0,04 \pm 0,03 \mu \mathrm{g} \cdot \mathrm{cm}^{-2}$ et de $9,76 \pm 2,35 \mu \mathrm{g}$. $\mathrm{cm}^{-2}$ à $0,11 \pm 0,09 \mu \mathrm{g} \cdot \mathrm{cm}^{-2}$ respectivement à des hauteurs de pulvérisation de $1 \mathrm{~m}$ et $1,5 \mathrm{~m}$. De même, la quantité moyenne de tartrazine enregistrée avec le pulvérisateur à dos pour chaque distance varie de $4,76 \pm 2,34 \mu \mathrm{g} \cdot \mathrm{cm}^{-2}$ à $0,03 \pm 0,02 \mu \mathrm{g} \cdot \mathrm{cm}^{-2}$ et de $5,82 \pm$ 


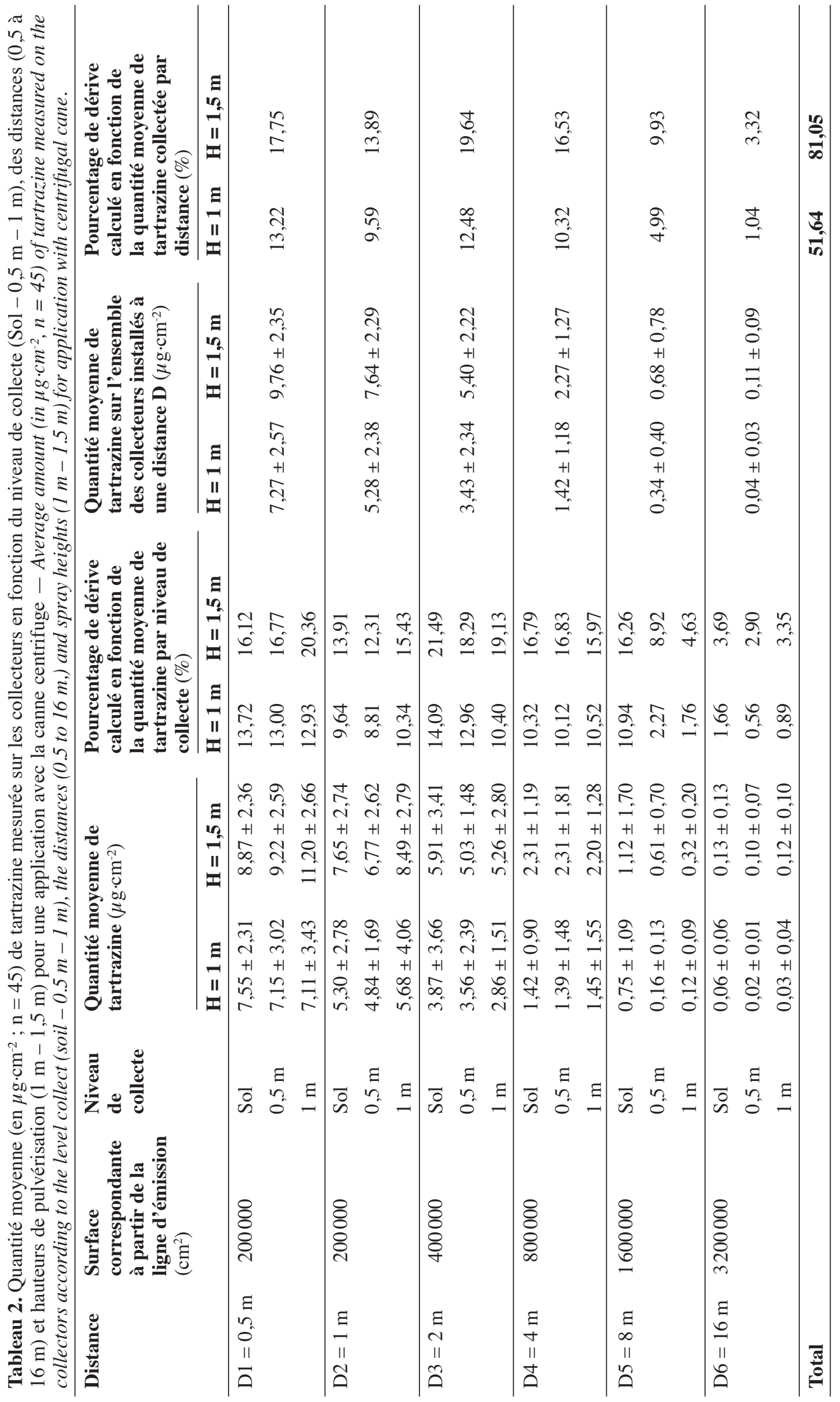




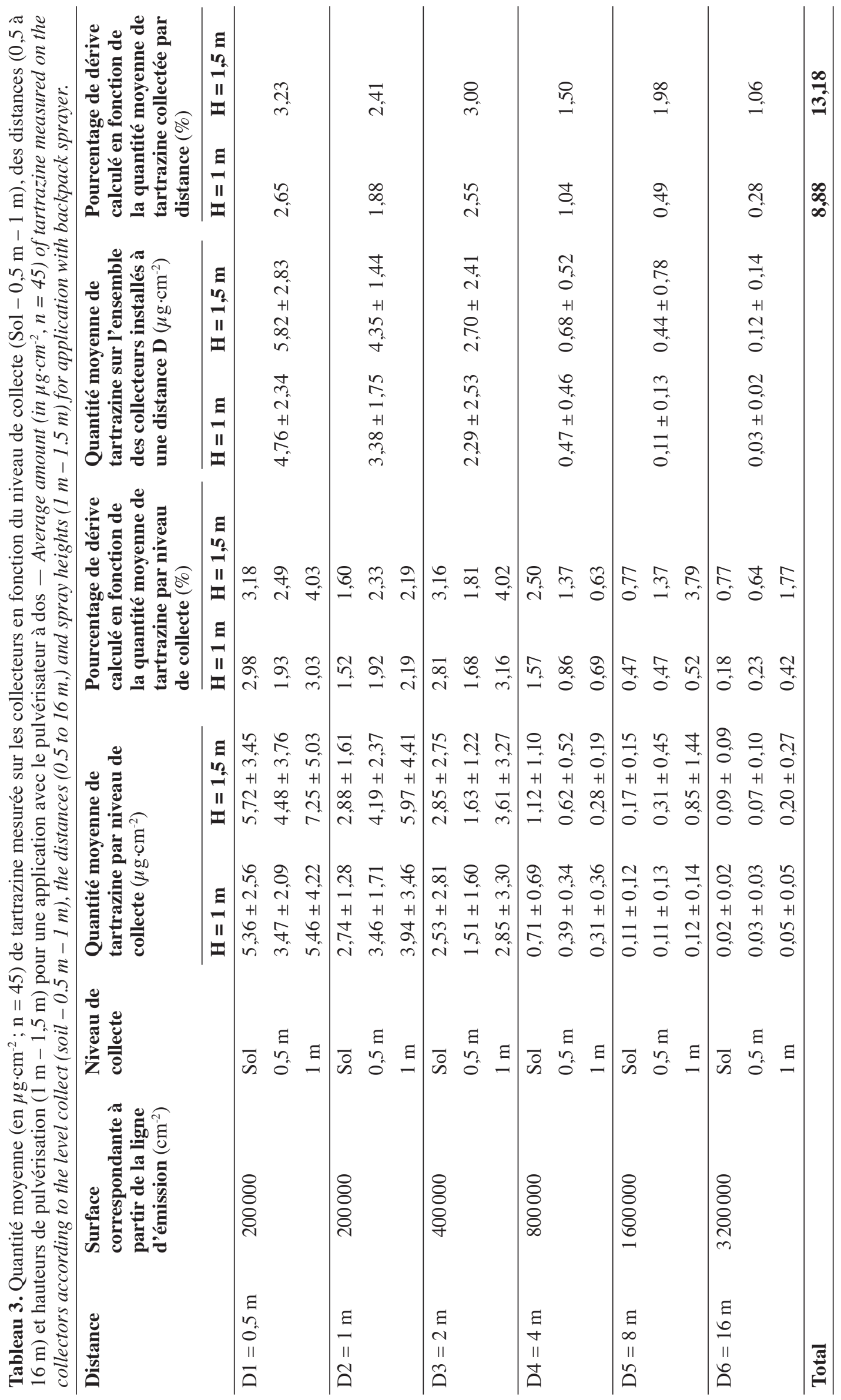


$2,83 \mu \mathrm{g} \cdot \mathrm{cm}^{-2}$ à $0,12 \pm 0,14 \mu \mathrm{g} \cdot \mathrm{cm}^{-2}$ respectivement pour des pulvérisations à $1 \mathrm{~m}$ et $1,5 \mathrm{~m}$ de hauteur.

À partir de la mesure des dépôts sur les collecteurs, le pourcentage de dérive est calculé par rapport à la masse totale de traceur pulvérisé, en multipliant la quantité par unité de surface déterminée au point de mesure par la surface entre les lignes de mesure. Pour chaque distance, et à chaque niveau de collecte, le pourcentage de dérive $(\% \mathrm{D})$ est ainsi déterminé suivant la formule :

$$
\% D=\frac{Q \times S}{M \times 100}
$$

où $D$ est le pourcentage de dérive à une distance donnée de la zone de pulvérisation (de 0,5 à $16 \mathrm{~m}$ ); $Q$ est la quantité moyenne de tartrazine par unité de surface (en $\mu \mathrm{g} \cdot \mathrm{cm}^{-2}$ ) au niveau de collecte ; $S$ est la surface correspondante à partir de la ligne d'émission $\left(\right.$ en $\mathrm{cm}^{2}$ ). La surface $S$ est calculée en faisant le produit de la longueur de la distance parcourue par l'opérateur $(40 \mathrm{~m})$ et la largeur entre les lignes de mesures ; $M$ est la masse de tartrazine $(\mu \mathrm{g})$ appliquée sur la surface traitée (en fonction du volume de bouillie mesuré : $M=11000000 \mu \mathrm{g}$ pour la canne centrifuge et $M=36000000 \mu \mathrm{g}$ pour le pulvérisateur à dos).

\subsection{Comparaison des dérives engendrées par la canne centrifuge et le pulvérisateur à dos}

La figure 2 permet de comparer les pourcentages de dérives engendrées par chacun des deux appareils en fonction des différentes hauteurs de pulvérisation $(\mathrm{H}=1 \mathrm{~m}$ et $\mathrm{H}=$ $1,5 \mathrm{~m})$ et suivant les distances par rapport au point d'émission (de $0,5$ à $16 \mathrm{~m})$.

\subsection{Analyse statistique des résultats}

Une analyse de la variance (à trois facteurs fixes: type de pulvérisateur; hauteur de pulvérisation; distance du point d'émission) sur l'ensemble des données montre qu'il existe une interaction entre le type de pulvérisateur et la distance $(p=$
$0,019<0,05)$. Le type de pulvérisateur utilisé a donc un effet sur la dérive. Les tests de comparaison de moyennes par ligne de collecte (avec comme facteurs fixes : type de pulvérisateur ; hauteur de pulvérisation) montrent qu'à $16 \mathrm{~m}$, la hauteur de pulvérisation a un effet sur la dérive, quel que soit le type d'appareil utilisé $(p=0,048)$. Les tests démontrent également qu'à $0,5 \mathrm{~m}$ $(p=0,012), 1 \mathrm{~m}(p=0,01)$ et $4 \mathrm{~m}(p=0,008)$, la dérive est supérieure avec la canne centrifuge. À partir de $8 \mathrm{~m}$, il n'y a plus de différence statistiquement significative entre les appareils.

\section{DISCUSSION}

De façon générale, la contamination des eaux dépend de la distance entre le lieu d'application et la masse d'eau (Orhon, 1993 ; Patty, 1997), les transferts se faisant par ruissellement ou par la dérive des gouttelettes. Selon Adechian et al. (2015), dans cette zone, $46 \%$ des producteurs ont un champ de coton situé à moins de $500 \mathrm{~m}$ d'un cours ou plan d'eau. En principe donc, avec un appareil tel que la canne centrifuge, le risque de contamination n'est pas négligeable car

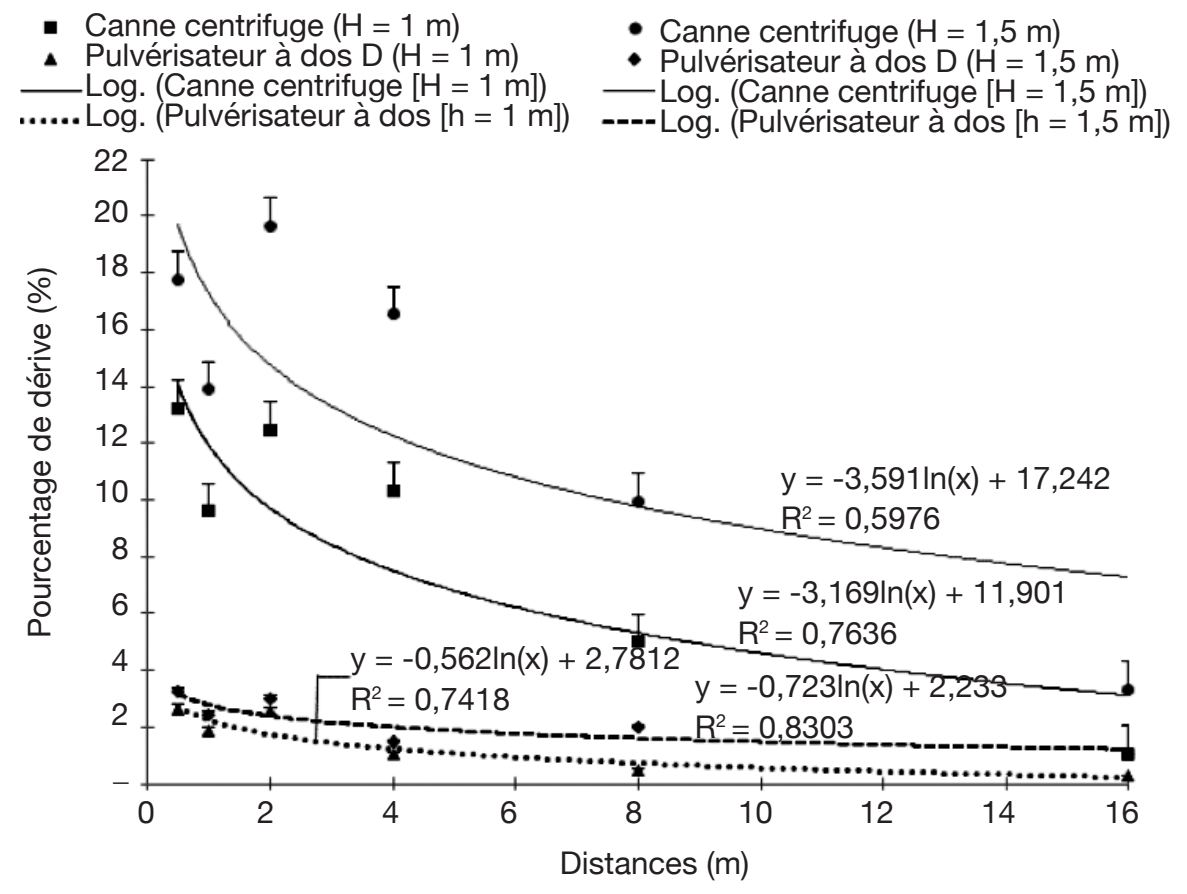

Figure 2. Comparaison des pourcentages de dérives engendrées par chacun des deux appareils en fonction des différentes hauteurs de pulvérisation $(\mathrm{H}=1 \mathrm{~m}$ et $\mathrm{H}=1,5 \mathrm{~m}$ ) et suivant les distances par rapport au point d'émission (de 0,5 à $16 \mathrm{~m}$ ). Les courbes de tendance sont ajustées par rapport aux points moyens calculés dans les tableaux $\mathbf{1}$ et $\mathbf{2}$ - Comparison of drift percentages generated by each of two spayers according to the different spray heights $(H=1 \mathrm{~m}$ and $H=1.5 \mathrm{~m})$ and the distances from the emission point $(0.5$ to $16 \mathrm{~m})$. The trend curves are adjusted compared to the average points calculated in tables 1 and 2. 
son fonctionnement est basé sur l'entrainement de la bouillie vers les rangs de cotonniers par le vent. Il était donc intéressant de quantifier cette dérive dans les conditions habituelles d'épandage par les paysans béninois.

Selon Gil \& Sinfort (2005), la dérive est influencée par les conditions météorologiques externes, dont la vitesse du vent. Avec une vitesse moyenne du vent plus ou moins constante lors des essais et égale à $3,0 \pm$ $0,4 \mathrm{~m} \cdot \mathrm{s}^{-1}\left(10,8 \pm 1,6 \mathrm{~km} \cdot \mathrm{h}^{-1}\right)$, l'examen des résultats indique des traces de tartrazine, hors de la zone ciblée par le traitement, jusqu'à une distance maximale de $16 \mathrm{~m}$ à partir du point d'émission. Or, dans le bassin cotonnier, les parcelles de cultures vivrières sont parfois juxtaposées à celles de coton, ce qui fait courir dans ce cas des risques d'intoxication alimentaire, car un transfert de bouillie sur des parcelles non ciblées est possible à cause des mouvements d'air (BCPC, 1986 ; Al Heidary et al., 2014).

Quel que soit le type de pulvérisateur utilisé et les hauteurs de pulvérisation ( $1 \mathrm{~m}$ et $1,5 \mathrm{~m}$ ), les quantités moyennes de tartrazine mesurées sur les collecteurs diminuent progressivement lorsqu'on s'éloigne du point d'émission (en passant de 0,5 à $16 \mathrm{~m}$ ). Les quantités maximales sont obtenues à $0,5 \mathrm{~m}$ du point d'émission $\left(9,76 \pm 2,35 \mu \mathrm{g} \cdot \mathrm{cm}^{-2}\right.$ et 5,82 $\left.\pm 2,83 \mu \mathrm{g} \cdot \mathrm{cm}^{-2}\right)$ et les quantités minimales $\left(0,11 \pm 0,09 \mu \mathrm{g} \cdot \mathrm{cm}^{-2}\right.$ et $0,03 \pm$ $0,02 \mu \mathrm{g} \cdot \mathrm{cm}^{-2}$ ) sont obtenues à $16 \mathrm{~m}$ respectivement avec la canne centrifuge et le pulvérisateur à dos La quantité de tartrazine obtenue par dérive lors des pulvérisations est donc inversement proportionnelle à la distance de collecte, quels que soient le type de pulvérisateur utilisé et la hauteur de pulvérisation. Ces résultats sont conformes à ceux obtenus par Stainier (2004), Bauwens (2008) et Nandjui (2009), lors des essais de quantification de dérive et de contamination.

Pour un même type d'appareil, à distance égale, les quantités de tartrazine obtenues sur les collecteurs placés au sol et à $1,5 \mathrm{~m}$ de hauteur de pulvérisation sont nettement supérieures à celles obtenues à $1 \mathrm{~m}$ de hauteur d'émission.

En ce qui concerne les dépôts au niveau du sol, une nette différence apparait entre les deux appareils. Pour la canne centrifuge, les quantités retrouvées au sol sont systématiquement plus élevées (+ 50 à $60 \%$ ) que les dépôts obtenus au sol avec le pulvérisateur à dos. En outre, quelle que soit la hauteur de pulvérisation, les quantités moyennes de tartrazine déposées sur les collecteurs placés au sol sont généralement supérieures à celles mesurées sur les collecteurs fixés à $0,5 \mathrm{~m}$ ou $1 \mathrm{~m}$ de hauteur. Pour cet appareil, la dérive est plus importante (de 10 à $20 \%$ ) et les plus grosses gouttes du spectre émis sédimentent rapidement, alors que la majorité de très fines gouttes est entrainée plus loin. Au contraire, pour le pulvérisateur à dos, la dérive est limitée (autour de 2 à $3 \%$ ) et les quantités au sol sont inférieures ou comparables à ce qui est collecté en hauteur.

En outre, les quantités maximales sont obtenues respectivement avec la canne centrifuge et le pulvérisateur à dos sur les collecteurs placés à $1 \mathrm{~m} \mathrm{du}$ $\operatorname{sol}\left(7,11 \pm 3,43 \mu \mathrm{g} \cdot \mathrm{cm}^{-2}\right.$ et $11,20 \pm 2,66 \mu \mathrm{g} \cdot \mathrm{cm}^{-2} ; 7,25 \pm$ $5,03 \mu \mathrm{g} \cdot \mathrm{cm}^{-2}$ et $\left.5,46 \pm 4,22 \mu \mathrm{g} \cdot \mathrm{cm}^{-2}\right)$, directement au sol $\left(7,55 \pm 2,31 \mu \mathrm{g} \cdot \mathrm{cm}^{-2}\right.$ et $8,87 \pm 2,36 \mu \mathrm{g} \cdot \mathrm{cm}^{-2} ; 5,72 \pm$ $3,45 \mu \mathrm{g} \cdot \mathrm{cm}^{-2}$ et $\left.5,36 \pm 2,56 \mu \mathrm{g} \cdot \mathrm{cm}^{-2}\right)$ et à $0,5 \mathrm{~m}(7,15 \pm$ $3,02 \mu \mathrm{g} \cdot \mathrm{cm}^{-2}$ et $9,22 \pm 2,59 \mu \mathrm{g} \cdot \mathrm{cm}^{-2} ; 4,48 \pm 3,76 \mu \mathrm{g}$. $\mathrm{cm}^{-2}$ et $\left.3,47 \pm 2,09 \mu \mathrm{g} \cdot \mathrm{cm}^{-2}\right)$. Traiter à plus grande hauteur conduit donc théoriquement à une perte plus importante du produit utilisé. Ces résultats corroborent ceux de Asman et al. (2003), Gil \& Sinfort (2005) et Baetens et al. (2009), qui ont aussi constaté que la hauteur de pulvérisation a une forte influence sur la répartition du produit lors des pulvérisations. Ils sont identiques à ceux de Siati (2005) qui explique cette différence à travers l'augmentation de la distance de chute et la vitesse du vent due à la variation de la hauteur; elle engendre la fragmentation des gouttelettes en de fines gouttelettes sensibles à la dérive (Matthews, 1992). On en déduit qu'en milieu réel, la dérive est donc plus importante lorsque, la taille du cotonnier augmentant, l'opérateur est obligé de soulever sa canne ou sa lance jusqu'au sommet de la végétation.

Pour les mêmes conditions météorologiques, une hauteur de pulvérisation identique et à distance égale, les pourcentages de dérive engendrés par la canne centrifuge sont nettement supérieurs à ceux obtenus avec le pulvérisateur à dos, quel que soit le niveau de collecte. Cela s'explique car le pulvérisateur à dos produit de plus grosses gouttelettes $(>150 \mu \mathrm{m})$ que la canne de pulvérisation centrifuge $(\leq 150 \mu \mathrm{m})$. Selon Salah et al. (2015), ces fines gouttelettes $(<150 \mu \mathrm{m})$, même si elles assurent une bonne rétention (Skuterud et al., 1988 ; Knoche, 1994), sont souvent sujettes au phénomène de dérive (Matthews, 1992) à cause de leur masse réduite ; elles sont plus sensibles aux forces de frottement aérodynamiques et de ce fait, livrées au mouvement de l'air et des conditions climatiques (Solie \& Alimardani, 1986 ; Qi et al., 2008 ; Bahrouni, 2010). Ces résultats confirment également ceux d'Aubertot et al. (2005) qui estiment que la dérive d'un pulvérisateur à dos peut varier de $1 \%$ à $10 \%$, en fonction des conditions météorologiques et de la hauteur d'application. Par contre, si l'on se réfère aux données de ces auteurs, le pourcentage de dérive observé pour la canne centrifuge est nettement supérieur à celui du pulvérisateur à rampe de buses. Il faut toutefois nuancer ces commentaires car les essais de dérive sont réalisés sur des terrains plats et en absence de végétation. En pratique, celle-ci pourrait intercepter une partie significative des gouttelettes.

La présence de végétation, la compétence des opérateurs, la rétention des substances au niveau du 
sol, ainsi que la dégradation physico-chimique ou biologique sont des paramètres qui vont pondérer les processus de transfert vers l'eau, le sol et l'atmosphère (Gbaguidi et al., 2011).

\section{CONCLUSIONS}

La canne de pulvérisation centrifuge et le pulvérisateur à dos sont les deux principaux types d'appareils utilisés pour les traitements phytosanitaires dans le bassin cotonnier au Bénin. Dans les mêmes conditions environnementales, la canne de pulvérisation centrifuge, qui est de loin l'appareil le plus utilisé par les producteurs (78 \% des producteurs de coton l'utilisent), génère une dérive supérieure à celle du pulvérisateur à dos. Cette tendance se renforce avec la hauteur de travail quand le cotonnier atteint sa croissance maximale. Hofman \& Solseng (2001) estiment en outre que l'opérateur peut favoriser ou limiter la dérive grâce à son expérience et sa compétence en agissant sur sa vitesse d'avancement pour réduire le temps de travail et sur la hauteur de pulvérisation de manière à ne pas laisser trop d'espace entre la buse et le cotonnier.

Si l'on considère, d'une part, ce que la dérive représente du pourcentage de la quantité appliquée au champ et, d'autre part, la distance entre la plupart des parcelles cultivées et la retenue d'eau, le risque de transfert des pesticides vers la surface des retenues d'eaux par voie aérienne semble limité. Par contre, le risque de contamination est réel pour les cultures vivrières contigües, exposant les consommateurs de ces produits aux résidus des insecticides particulièrement toxiques épandus sur coton.

\section{Remerciements}

Les auteurs tiennent à remercier l'ARES pour le soutien financier au projet de recherche AQUATOX.

\section{Bibliographie}

Adechian A.S. et al., 2015. Les pratiques paysannes de gestion des pesticides sur le maïs et le coton dans le bassin cotonnier du Bénin. VertigO, 15(2).

Agbohessi P.T. et al., 2011. Caractérisation des pesticides chimiques utilisés en production cotonnière et impact sur les indicateurs économiques dans la Commune de Banikoara au nord du Bénin. Int. J. Biol. Chem. Sci., 5(5), 1828-1841.

Agbohessi T.P., 2014. Impact des pesticides agricoles sur les performances de reproduction des poissons dans le bassin cotonnier béninois. Thèse de doctorat : Université de Namur (Belgique).

AIC (Association Interprofessionnelle du Coton), 2005. L'interprofession, rôle, enjeux et perspectives. In :
Communication présentée à la Conférence Coton de l'Afrique de l'Ouest et du Centre, 10-12 mai 2005, Cotonou, Bénin .

Al Heidary M., Douzals J.P., Sinfort C. \& Vallet A., 2014. Influence of spray characteristics on potential spray drift of field crop sprayers: a literature review. Crop Prot., 63, 120-130.

Asman W., Jørgensen A. \& Jensen P., 2003. Dry deposition and spray drift of pesticides to nearby water bodies. Copenhague: Danish EPA.

Aubertot J.N. et al., eds, 2005. Pesticides, agriculture et environnement. Réduire l'utilisation des pesticides et limiter leurs impacts environnementaux. INRA/ CEMAGREF.

Baetens K., 2009. A validated 2-D diffusion-advection model for prediction of drift from ground boom sprayers. Atmos. Environ., 43, 1674-1682.

Bahrouni H., 2010. Caractérisation de l'efficacité technique des systèmes de pulvérisation et des pertes de pesticides appliqués aux cultures basses dans les régions méditerranéennes : cas de la Tunisie. Thèse de doctorat: Université de Carthage (Tunisie) et Montpellier Supagro (France).

Bauwens A., 2008. Modélisation de la dérive d'un pulvérisateur à partir de mesures effectuées par capteurs embarqués. Mémoire : Faculté universitaire des Sciences agronomiques de Gembloux (Belgique).

BCPC (British Crop Protection Council), 1986. Nozzle classification scheme. Alton, UK: BCPC.

Bonicelli B. et al., 2012. Mesure en continu de la qualité de l'air en aval d'une zone de traitement. In : $42^{e}$ Congrès du Groupe Français des Pesticides, mai 2012, Poitiers, France.

Briand O., Bertrand F., Seux R. \& Millet M., 2001. Comparison of different sampling techniques for the evaluation of pesticide drift in apple orchards. Sci. Total Environ., 288(2002), 199-213.

Bylemans D., 2001. The importance of application technique and drift reduction for ecotoxicological risk assessment and risk management in fruit growing. Parasitica, 57(12-3), 5-11.

Celini L., 2001. Le puceron du cotonnier. Aphis gossypii (Glover) et son parasite Aphelinus gossypii Timberlake en République centrafricaine. Insectes, 122(3), 710.

Dembélé K., 2006. Étude socio-économique de l'utilisation des pesticides chimiques de synthèse. Cas de la filière coton au Mali. Mémoire: Faculté universitaire des Sciences agronomiques de Gembloux (Belgique).

Djihinto C.A., Affokpon A., Dannon E. \& Aboua C., 2016. Réduction de doses de cyperméthrine-triazophos et lutte raisonnée en culture cotonnière au Bénin. J. Appl. Biosci., 98, 9261-9269.

Doumbia M. \& Kwadjo K.E., 2009. Pratiques d'utilisation et de gestion des pesticides par les maraîchers en Côte d'Ivoire: cas de la ville d'Abidjan et deux de ses 
banlieues (Dabou et Anyama). J. Appl. Biosci., 18, 9921002.

Eddaya T. et al., 2015. Utilisation et risque des pesticides en protection sanitaire de la menthe verte dans le Centre-Sud du Maroc (Use and risks of pesticides in sanitary protection spearmint in south-central Morocco). J. Mater. Environ. Sci., 6(3), 656-665.

Gbaguidi M.A.N. et al., 2011. Évaluation quantitative des résidus de pyréthrinoïdes, d'aminophosphate et de triazines en zones de production de coton au Bénin par la méthode ELISA en phase liquide : cas des eaux de la rivière Agbado. Int. J. Biol. Chem. Sci., 5(4), 1476-1490.

Gil Y. \& Sinfort C., 2005. Emission of pesticides to the air during sprayer application: a bibliographic review. Atmos. Environ., 39, 5183-5193.

Gil Y., 2007. Caractérisation expérimentale des émissions de pesticides vers l'air pendant les pulvérisations viticoles. Thèse de doctorat: Montpellier SupAgro (France).

Herbst A., 2001. A method to determine spray drift potential from nozzles and its link to buffer zone restrictions. St. Joseph, MI, USA: American Society of Agricultural and Biological Engineers.

Hofman V. \& Solseng E., 2001. Reducing spray drift. Agricultural and Biosystems Engineering. Fargo, ND, USA: North Dakota State University.

Kanda M., 2011. Agriculture maraîchère au Togo : analyse systémique et environnementale. Thèse de doctorat: Université de Lomé (Togo).

Katé S. et al., 2014. Perceptions locales de la manifestation des changements climatiques et mesures d'adaptation dans la gestion de la fertilité des sols dans la Commune de Banikoara au Nord-Bénin. J. Appl. Biosci., 82, 74187435.

Knoche M., 1994. Effect of droplet size and carrier volume on performance of foliage-applied herbicides. Crop Prot., 13, 163-178.

Kodjo E.A., 2007. ANCE fights for the prohibition of the use of endosulfan in Togo. International POPs Elimination Network (IPEN).

Lawson A.J., Akohou H., Lorge S. \& Schiffers B., 2017. Three methods to assess levels of farmers' exposure to pesticides in the urban and peri-urban areas of Northern Benin. Tunis. J. Plant Prot., 12(1), 91-108.

MAEP (Ministère de l'Agriculture, de l'Élevage et de la Pêche), 2016. Rapport d'activités - Définition d'un nouveau cadre institutionnel de gestion de la filière coton au Bénin (Document technique de réflexion). Cotonou : MAEP.

Matthess A., Van den Akker E., Chougourou D. \& Midingoyi G.S., 2005. Compétitivité et durabilité de cinq systèmes culturaux cotonniers dans le cadre de la filière. Cotonou : ProCGRN/GTZ et MAEP.

Matthews G.A., 1992. Pesticide application methods. $2^{\text {nd }} e d$. Harlow, UK: Longman.

Miller P.C.H. \& Butler Ellis M.C., 2000. Effects of formulation on spray nozzle performance for applications from ground-based boom sprayer. Crop Prot., 19, 609615.

Murray R.A., Cross J.V. \& Ridout M.S., 2000. The measurement of multiple spray deposits by sequential application of metal chelate tracers. Ann. Appl. Biol., 137, 245-252.

Nandjui J., 2009. Quantification de la dérive en conditions réelles. Mémoire: Faculté universitaire des Sciences agronomiques de Gembloux (Belgique).

Okoumassoun L.E. et al., 2002. Vitellogenin in tilapia male fishes exposed to organochlorine pesticides in Ouémé River in Republic of Benin. Sci. Total Environ., 299, 163-172.

Orhon L., 1993. Influence de la distance des épandages au ruisseau collecteur sur le transfert par ruissellement des pesticides. Mémoire de DEA : École Nationale de Santé Publique, Rennes (France).

Patty L., 1997. Limitation du transfert par ruissellement vers les eaux superficielles de deux herbicides (isoproturon et diflufénicanil). Méthodologie analytique et étude de l'efficacité de bandes enherbées. Thèse de doctorat: Université Joseph Fourier (Grenoble).

Pazou E.Y.A. et al., 2006a. Organochlorine and organophosphorous pesticide residues in the Ouémé river catchment in the Republic of Benin. Environ. Int., 32, 616-623.

Pazou E.Y.A. et al., 2006b. Contamination of fish by organochlorine pesticide residues in the Ouémé river catchment in the Republic of Bénin. Environ. Int., 32, 594-599.

Qi L., Miller P.C.H. \& Fu Z., 2008. The classification of the drift risk of sprays produced by spinning discs based on wind tunnel measurements. Biosyst. Eng., 100, 38-43.

Rachadi T., 2010. Manuel de lutte antiacridienne. Versailles, France : Quæ \& CTA.

Reichenberger S., Bach M., Skitschak A. \& Frede H.G., 2007. Mitigation strategies to reduce pesticide inputs into ground- and surface water and their effectiveness. A review. Sci. Total Environ., 384, 1-35.

Salah S.O.T. et al., 2015. Étude des potentialités des systèmes d'application contrôlée des gouttes (CDA) pour les traitements phytosanitaires en céréaliculture (synthèse bibliographique). Biotechnol. Agron. Soc. Environ., 20(S1), 287-298.

Sanborn M. et al., 2004. Pesticides literature review. Toronto, Canada: Ontario College of Family Physicians.

Schiffers B., 2011. Fondements de la protection des cultures. Manuel $n^{\circ} 7$. Bruxelles : Programme PIP/COLEACP.

Schilter C., 1991. L'agriculture urbaine à Lomé. Approches agronomiques et socio-économiques. Paris : IUED, Karthala.

Siati M., 2005. Étude de quelques paramètres permettant une évaluation et une réduction des risques de dérive lors de l'application des traitements phytosanitaires : cas de pulvérisateur à dos. Mémoire : Faculté universitaire des Sciences agronomiques de Gembloux (Belgique). 
Skuterud R., Nordby A. \& Tyldum A., 1988. Effects of application methods, spray volumes, pressures and herbicide rates on weed control in spring cereals. Crop Prot., 7, 303-308.

Solie J.B. \& Alimardani R., 1986. Predicting spray patterns of a vertical rotary disc atomizer. Trans. ASAE, 29(5), 1193-1198.

Somerhausen E., 2006. Les mesures de réduction de la dérive lors des traitements phytosanitaires, http://pro. ovh.net/ fiwap/uploads/File/Bibliotheques/Technique/ Pulveetprodphyto/0606reductionderivepulverisation. pdf, (27/04/2018).

Sougnabe S.P. et al., 2009. Pratiques phytosanitaires paysannes dans les savanes d'Afrique centrale. In : Actes du Congrès, Savanes africaines en développement: innover pour durer, 20-23 avril 2009, Garoua, Cameroun, 1-13.

Stainier C., 2004. Influence des propriétés de mélanges phytosanitaires sur la dérive générée par trois types de buse de pulvérisation. Mémoire: Faculté universitaire des Sciences agronomiques de Gembloux (Belgique).
Thiam A. \& Sagna M.B., 2009. Monitoring des pesticides au niveau des communautés à la base. Rapport Régional Afrique. Dakar, Sénégal: Pesticide Action Network Africa.

Toé A.M., Kinane M.L., Kone S. \& Sanfo-Boyarm E., 2004. Le non-respect des bonnes pratiques agricoles dans l'utilisation de l'endosulfan comme insecticide en culture cotonnière au Burkina Faso: quelques conséquences pour la santé humaine et l'environnement. Rev. Afr. Santé Prod. An., 2(3-4), 275-278.

Traoré S.K. et al., 2006. Contamination de l'eau souterraine par les pesticides en régions agricoles en Côte d'Ivoire (Centre, Sud et Sud-Ouest). J. Afr. Sci. Environ., 1, 1-9.

Zoumenou B. et al., 2015. Effets toxicologiques et méthodes d'analyse de la lambda-cyhalothrine et de l'acétamipride utilisés dans la protection phytosanitaire du cotonnier au Bénin. Int. J. Biol. Chem. Sci., 9(4), 2184-2199.

(57 réf.) 